\title{
Research and Applications of Cloud Desktop*
}

\author{
Liu Xia \\ Sanya Aviation and Tourism College, Sanya 572000, China \\ liuxia83@vip.qq.com
}

\begin{abstract}
With the development of information technology, enterprises are increasingly challenged in internal IT equipment management. A huge number of PC devices made enterprises' daily management of employees' personal computer a complex task, and enterprises often need to invest lots of manpower and resources in upgrades, security policy, and routine equipment maintenance. While with workplaces scattered, mobile devices began to be used in office, enterprises are faced with the challenge of mobile office, and the traditional PC devices cannot fully meet the office needs of enterprises. The concept of cloud computing has risen in recent years; cloud desktop, this new form of office equipment, also came into people's view. As an extension of virtualization, cloud desktop, which relies on server-side computing, has high safety, easy management, and is suitable for mobile devices. Cloud Desktop has already had a large number of application cases at home and abroad, pointing a new way for IT office equipment selection.
\end{abstract}

Index Terms - Cloud computing, desktop virtualization, application, prospects

\section{Introduction}

Cloud computing has been a hot topic in IT area since it came into being in 2007. Cloud computing[1], IBM white paper, and professor LiuPeng[2] both have defined on it

Desktop virtualization is a coupling of the desktop environment of users and the terminal devices that he or she uses. What is stored in the server is each user's full desktop environment, and they can visit it through internet by using different terminal devices which are of processing and displaying function[3].

\section{A Review of Cloud Desktop}

\section{A.Definition}

Cloud Desktop is a reflection of Cloud Computing on the front end, it is further expressed in visiting cross-platform applications through thin client device or any other devices which are connected to the internet[4].

Nowadays almost all cloud computing vendors have businesses in cloud desktop, but their business names are different. Some of them call it Cloud Desktop, while some call it Virtualized Desktop. IBM described its IBM Smart Business Desktop Cloud like this: You can visit cross-platform applications and the entire client desktop through thin client device or any other devices which are connected to the internet. Although other vendors have different names for their services, their specific business is the same as IBM.

We can see that Cloud Desktop is a kind of computing mode which is centered on server. You can visit personal desktop which resides on server side and various applications through specific procedures or browsers, only with a small carton-sized client terminal, or any other devices that can be connected to the internet, and its user experience is the same as that of using traditional PC.

\section{B. Advantages and Disadvantages of Cloud Desktop}

\section{1) Advantages}

Low overall cost: The cost of IT asset is composed of many aspects, the hard cost of buying equipment in the early days accounts for only a small part of the overall cost, besides that, the management, maintenance of equipment, the cost caused by energy consumption, and the cost of hardware upgrade over the life cycle of the equipment are also parts of overall cost. Compared with traditional office equipment-PC, Cloud Desktop reduced the soft cost over the life cycle greatly. Because of its low soft cost and the large proportion of soft cost in overall cost, so the overall cost of Cloud Desktop is lower.

Information Safety: Because there are no operating systems and applications on the thin client, the threat of virus is extremely low, and the access equipment can be controlled, which reduces the threat caused by external storage of users greatly. Besides that, because of the one-to-one relationship between the desktop and the user, each operation can be traced back to a specific user, which is good for locating the source of problems.

Easy management: The Cloud Desktop provides unified maintenance for users' desktop, which realized unified patch loading, unified virus library upgrading, and unified application management, these reduce artificial workloads greatly, and because thin client is adopted, the maintenance of terminal is almost zero.

Mobile office: Users can visit their own desktops from any terminal. They can access it with various terminals at anytime and anywhere: on the desk, in the office, on the trip, or at home. This made the one-to-one relationship of desktop resources and user permission possible. All data and desktops are operated and saved together on the data center, users can change terminal of hot plugging without suspending applications.

Energy conservation and environmental protection: The power of thin client is at around 10W, which is far below the traditional power consumption: $200-300 \mathrm{~W}$, this can reduce energy consumption effectively. When the thin client is operating, its operating temperature is low and there is no noise. This can solve the problem of temperature and noise in

\footnotetext{
* This work is partially supported by A cooperative science project between colleges and local government in Sanya\#2013YD64 to LiuXia
} 
intensive office environment like call center when it is deployed close human beings.

\section{2) Disadvantages}

High hardware cost: In the initial process of the construction of the Cloud Desktop, equipments like server, storage device and network device should be bought. Although the price of thin client is much lower than that of traditional PC, the cost of background hardware like server and storage cover the advantage of purchasing price of Cloud Desktop project in the early days.

High demand of network environment: The Cloud Desktop project is a kind of server-side computing, which means the operation and stored data interaction between subscriber terminal and server side are totally relied on internet. Once there is something wrong with the network transmission contact, all users who access the server through this contact will be affected, which brings inconvenience to daily work.

High safety risk of the server: All terminal operation and data storage of users rely on network terminal server. If the performance of the network terminal server is insufficient or crash, all daily work of users burdened by this terminal server will be affected, that is to say, if there is something wrong with one terminal server, a large number of users will be affected negatively with work inconvenience.

\section{The current situation and trends of the Cloud Desktop}

As the embodiment of cloud computing, traditional server virtual technology facilitators like Vmware, Citrix, and Microsoft have all launched solutions for Cloud Desktop. IBM, HP, SUN and many other major companies have also put a lot into Cloud Desktop. For example, the solution of the Smart Business Desktop Cloud Computing put forward by IBM, and Sunray launched by SUN. The Cloud Desktop has been a hot topic in the field of technology, and each major manufacturer will continue to introduce mature and overall solutions $^{[5]-[9]}$.

Besides that, more and more companies are paying attention to and investing the Cloud Desktop. It can not only meet the need of specific field like business lobby and call center, but also can provide services for public places like middle and small-sized enterprises, domestic consumers, hotels and airports, its range of target user is extremely wide. At present, some communication service providers, banks, and technology developers have used the Cloud Desktop in largescale inside their offices. For example, Huawei, a world wellknown communication provider, built the largest Cloud Desktop system in the world inside their offices, which can be used by 70,000 people at the same time.

With the advances in energy conservation and emission reduction, and the increase for the demand of enterprise security and secrecy, and IT cost control, the Cloud Desktop will also be increasingly concerned and favored by users, and entering an explosive development stage, becoming the heavyweight product in cloud computing.

\section{The Application Cases of Cloud Desktop}

\section{1) SH research institute of Huawei}

Huawei has begun to deploy Cloud Desktop in its $\mathrm{SH}$ research institute since 2009. There are 10,000 employees in its SH research institute, around 8,000 of them are RD (research and development) technician, whose main job is to research and develop the skills and products like wireless and core network. Staffs can connect the virtual server in the data center to deal with daily work at anytime and anywhere with only typing their account numbers and codes.

Through adopting the Cloud Desktop system, Huawei saved a lot of resources for SH research institute: for previous cost of hardware deployment along, it saved $40 \%$, and it also saved electricity charge $71 \%$. The system deployment cycle was shortened from 3 months to less than one week; and the maintenance personnel for IT was simplified from 200 to 9 .

\section{2) The Cloud Desktop of Shanghai Unicom}

The first phase of the Cloud Desktop project of Shanghai Unicom covered 5,000 users, and it can support any kinds of requirements for peripherals at the business hall outlets, for example, printers, scanners, fingerprints, card readers, headsets and so on. Most of IT applications in Shanghai Unicom, including its office system OA, production system OSS, BSS and so on, will all be transferred to Cloud Desktop, and eventually, the machine can be separated from human beings. In Shanghai Unicom, every employee can access the virtualized operating system at the cloud platform quickly; no matter in what kinds of network environment, and what they need is only an ID card and its corresponding password. By the end of 2011, all call centers' desktops in Shanghai Unicom will have been virtualized and $80 \%$ of business halls will be installed with thin terminal.

Apart from increasing the work efficiency internally, the Cloud Desktop also helped Shanghai Unicom build a brandnew desktop rental business mode. During working hour, the Cloud Desktop provided the business hall, call center and OA applications of Shanghai Unicom with resources using on demand; while in off hours, the Cloud Desktop background server provided computing resource rental service for 3D render enterprises like rock crystal firm and so on. Now, the Cloud Desktop has been the benchmarking project for Shanghai Unicom to build a smart city during the 12th FiveYear Plan.

\section{An analysis of Enterprise Cloud Desktop Development}

\section{A. Business Requirements of Cloud Desktop}

Pressure of maintenance cost: In daily work, a great deal of manpower should be put into management and maintenance. At the same time, because PC consumes much power, enterprises are under great pressure of IT cost.

Requirements for information safety: With more and more confidential information in enterprise is processed through PC, the risk of information safety is also increased. The traditional office equipment-PC can hardly monitor users efficiently, enterprises is confronted with risks of being 
attacked by external staffs to obtain confidential information through internet. In its overall construction, enterprises need to improve their protection ability of information safety constantly.

Requirements for mobile office: With the development of mobile technology, more and more people in the enterprise began to deal with daily office work with cell phone and tablet PC. Because of the great number of office addresses, employees also need to work in another place. The Cloud Desktop technology can meet these needs perfectly.

Requirements for construction of application places: The typical application scenarios of the Cloud Desktop include common office, research and development office, mobile office, call center, business hall, branches, task-oriented office, high-performance graphics, high-security desktop, multi-tenant operation and so on. It almost covered all scenarios for IT equipment of daily office in the enterprise. If traditional PC can be replaced by the Cloud Desktop, on one hand, the overall cost can be reduced; on the other hand, the working environment can also be improved.

\section{B. The Business Challenge Faced by Enterprise Cloud Desktop}

Construction cost in the early days: Although the overall cost of the Cloud Desktop is lower than that of PC, the initial cost of hardware and software is higher than that of traditional equipment. And the high construction cost in the early days is not good for enterprise to control the operating cost.

Earning cycle: The Cloud Desktop is high in construction cost in the early days and low in maintenance cost later on, which takes 2 years to show the advantage of lower overall cost. And it should be used for a relatively long time if you want to obtain application benefits.

Web environment: Because the Cloud Desktop has a high demand for web environment, it is mainly deployed through regional LAN in the industry. If the Cloud Desktop is deployed in a large scale in the industry, the web environment should be optimized and expanded depending on specific situations. And this will cause a raise in web cost objectively.

The using habit of users: The enterprise employees are accustomed to using PC to deal with office work, and all documents and data are saved in local device. While in Cloud Desktop, all documents and data are saved in server side, and it takes time for users to adapt to the change of data storage location, at the same time, the transfer of existing PC data will also bring a certain cost.

Apart from the strengths of Cloud Desktop like safety, easy management and so on, enterprises still need to solve various construction challenges in its internal applications according to the specific situations.

\section{An Analysis of Revenue and Cost in Enterprise Cloud Desktop}

A big advantage of the Cloud Desktop is its low overall cost. Although its initial hardware and software cost is higher that of PC, it accounts for less than $30 \%$ in the overall cost. While the end-user charge in later days accounts for nearly
$50 \%$ in the overall cost. It is through reducing operating cost and end-user charge to reduce the overall cost of Cloud Desktop and obtain application benefit.

With the continuous development of hardware technology, the price of traditional PC becomes lower and lower. At present, the price of a PC which can meet the daily office demands is around 4,000 Yuan, in which the cost of operating system has been included. While the average cost of a Cloud Desktop is nearly 6,000 Yuan, because it also involves the cost of server, storage, software permission and manually debugging.

Even though the direct cost of the Cloud Desktop is higher than that of traditional PC, it has advantages in time cost of software installation, maintenance cost, and energyconsumption cost and so on.

Take 2,000 Cloud Desktops as an example, the Cloud Desktop can save 1833.4 hours in the installation of related software and debugging, thus a labor cost of 1,480,000 Yuan can be saved.

It takes only 15 working staffs to maintain the 2,000 Cloud Desktops, while it takes about 40 people to maintain the PC of the same size. We assume that the annual pay of maintenance personnel is 60,000 Yuan; so a labor cost of around 1,500,000 Yuan can be saved one year.

Even if we split the energy consumed by devices like server and storage, the average energy consumption of Cloud Desktop is only $20 \%$ of that of traditional PC. Take the electricity price as 1 Yuan per kilowatt, 2,000 Cloud Desktops can save electricity costs of around 760,000 Yuan per year.

Because the hardware, software and the time cost of initial installation are single input, while the cost of labor maintenance and electricity are increased year by year. Through the previous cost data, it can be worked out that the cost advantage of Cloud Desktop can be showed in the second year after it is put into use, and the application benefits are also obtained at this time.

Generally speaking, the service cycle of a PC is about 4 year. In an entire service cycle, the overall cost of PC is much higher that of Cloud Desktop. In that case, 2,000 Cloud Desktop applications can bring an application benefit of about 4,000,000 Yuan.

Based on above analysis, in spite of the high construction cost of Cloud Desktop in the early days, its cost advantage in labor maintenance and energy-consumption make the overall cost of Cloud Desktop is lower that of PC in the 4-year application period. It takes 1.5 year for Cloud Desktop to show its cost advantage after it is put into use. And the longer it is used, the greater benefits which result from reducing cost it will get.

\section{Suggestions for the Development of Enterprise Cloud Desktop}

\section{A. Spread the concept of overall cost}

The biggest challenge faced by Cloud Desktop is the high construction cost in the early days. Although it has many advantages, and its overall cost is lower than that of traditional 
PC, these advantages are concentrated in invisible factors, for example, information safety, easy maintenance and so on. Traditional enterprises cannot find its advantages easily, and on the contrary, in the most direct initial purchase price, the price of Cloud Desktop is higher than that of PC. The price of traditional PC becomes lower and lower, while the Cloud Desktop project needs higher hardware and software purchasing price, which cannot be accepted by most enterprises.

To develop the Cloud Desktop, a major preoccupation is propaganda. The overall advantages and disadvantages of Cloud Desktop should be propagandized objectively and comprehensively, making every enterprise realize the real value of Cloud Desktop. At the same time, spread the concept of overall cost, then enterprises will not merely consider the initial direct purchase cost when choosing IT equipment, the maintenance cost and energy-consumption cost in later days should also be took into consideration, reducing the IT equipment charge from an overall view. Only through spreading the concept of overall cost can enterprises change the overall impression for Cloud Desktop, and accept the application of Cloud Desktop.

\section{B. Deploy Cloud Desktop around LAN}

The Cloud Desktop technology depends on web environment seriously, according to an analysis of existing Cloud Desktop use cases; the web environment will influence the overall judgments of users to Cloud Desktop directly. The Cloud Desktop is most likely to affect user experience like being stuck or stopped; actually, most of these cases are a result of web obstruction. So, if enterprises plan to construct Cloud Desktop in a large scale, a high-quality overall web environment is necessary.

Because of the technological feature of depending on internet, a large scale of Cloud Desktop deployment in industry is applied in LAN and internal network environment. For the few wide area network applications, technology vendors also developed network acceleration technology to ensure user experience. Enterprises should combine their existing basic network pattern when carrying out the construction of Cloud Desktop, centered on existing web environment of data center and office building, with the internal internet as the main body, to ensure the construction of the web environment of Cloud Desktop, and the service quality of Cloud Desktop.

\section{Replace Traditional PC in Batches}

The average cost of Cloud Desktop equipment is higher than that of PC, and PC has been used extensively as the main office equipment in the enterprise. If PC were directly replaced by Cloud Desktop, a huge alternative cost will be caused, thus increasing the operating pressure of enterprises.

A feasible approach to replace PC is to replace it with Cloud Desktop in batches. Firstly, to improve the information safety guarantee grade, applying Cloud Desktop in office system which has a high demand for information safety, for example, financial system, laws and regulations system and so on; then for traditional office application needs, it is in office area of appropriate web environment apply Cloud Desktop, spreading the concept of Cloud Desktop through experimental units. For typical Cloud Desktop application scenarios like newly-built call center, using the Cloud Desktop directly to reduce the overall construction cost. Through replacing PC gradually with Cloud Desktop in various batches, enterprises can no longer purchase traditional PC, and eventually, the Cloud Desktop can be used as the main IT equipment.

\section{Conclusion}

As a specific embodiment of cloud computing, the Cloud Desktop can reduce the IT cost of enterprises effectively. It has advantages of safety, easy management, energy conservation and environmental protection, low overall cost, and at the same time, it can also meet the needs of mobile office. There have been many application cases of Cloud Desktop in the world; it is a technological trend to replace PC with Cloud Desktop for enterprises which have numerous PC equipments. By applying Cloud Desktop, enterprises can not only reduce the cost of IT equipment, but also can improve the protection levels of information safety, reduce energyconsumption of enterprises, at last, increasing the overall working efficiency with new technology.

\section{References}

[1] http://download.boulder.ibm.com/ibmdl/pub/softeware/dw/wes/hipods/cl oud_computing_wp_final_8oct.pdf

[2] Liu Peng, 'Cloud Computing', 2nd Ed., Electronics Industry Press, 2011, BeiJing.

[3] Cheng Jingjing, 'Research and design of cloud desktop technology scheme based on Virtualization', Guangdong Communication Technology, no, 6, pp. 36-39, June 2011.

[4] http://baike.baidu.com/view/6497595.htm

[5] Feng Dengguo, Zhang Min, Zhang yan, Xu Zhen 'Study on Cloud Computing Security', Journal of Software, vol.22,no.1,pp. 71-83, November 2010.

[6] http://www900.ibm.com/ibm/ideasfromibm/cn/cloud/solutions/index.shtml

[7] http://developers.sun.com.cn/blog/functionalca/resource/sun 353cloudcomputing_chinese.pdf

[8] Barroso LA, Dean J, Holzle U. 'Web search for a planet: The Google cluster architecture'. IEEE Micro, no.2,pp.22-28,2003

[9] Chen Kang, Zheng Weimin. 'Cloud Computing :System Instances and Current Research', Journal of Software,vol.20,no.5, pp. 13371348, May 2009 